\title{
A STUDY OF CO-PLANING TECHNOLOGY OF SPACEBORNE, AIRBORNE AND GROUND REMOTE SENSING DETECTING RESOURCE, DRIVEN BY DISASTER EMERGENCY TASK
}

\author{
Fei Yu ${ }^{1}$, Hua Chen ${ }^{1}$, Kuan Tu ${ }^{1}$, Qiang Wen ${ }^{1,}{ }^{*}$, Jianjun He ${ }^{1}$, Xinzhi Gu ${ }^{1}$, Zhiyong Wang ${ }^{2}$ \\ ${ }^{1}$ Twenty First Century Aerospace Technology Co., Ltd., Beijing, China - (yufei, shenhua, tukuan, wenqiang, hejj, guxz) \\ @ 21at.com.cn \\ ${ }^{2}$ Beijing Engineering Research Center of Small Satellite Remote Sensing Information, Beijing, China - wangzy@21at.com.cn
}

Commission III, ICWG III/Iva

KEY WORDS: Disaster Emergency, Co-planning, Observation Resources, Mission-driven

\begin{abstract}
:
Facing the monitoring needs of emergency responses to major disasters, combining the disaster information acquired at the first time after the disaster and the dynamic simulation result of the disaster chain evolution process, the overall plan for coordinated planning of spaceborne, airborne and ground observation resources have been designed. Based on the analysis of the characteristics of major disaster observation tasks, the key technologies of spaceborne, airborne and ground collaborative observation project are studied. For different disaster response levels, the corresponding workflow tasks are designed. On the basis of satisfying different types of disaster monitoring demands, the existing multi-satellite collaborative observation planning algorithms are compared, analyzed, and optimized.
\end{abstract}

\section{INTRODUCTION}

Frequent occurrences of major hydrology, meteorology disaster and great earthquake have brought serious threats to people's lives, property and safety. In order to achieve rapid monitoring, accurate evaluation and efficient decision-making of disaster emergency response, the key techniques of spaceborne, airborne and ground remote sensing detecting resource collaborative observation integration and efficient application are urgently needed. It is necessary to develop the quick emergency response capability of spaceborne, airborne and ground remote sensing united from surface to terrestrial space. With the rapid development of earth observation field, cooperative planning and scheduling of satellites is faced with many problems, such as enormous task, diverse of payloads, poor scalability, complex constraints and low timeliness. At the same time, in order to meet the needs of severe disasters, it is urgent to carry out the collaborative planning technology of spaceborne, airborne and ground monitoring resources driven by disaster emergency task.

At present, in the field of satellite task planning and scheduling, the routine tasks of single-type observation resources are concerned at home and abroad. For example, researchers have proposed a variety of models and algorithms to solve satellite observation scheduling problems. Task scheduling models include linear model (Gabrel, 2006), constraint satisfaction model (Frank et al., 2000), weighted constraint model (Verfaillie et al., 1996), graph model (Gabrel et al., 2002), etc. Model solution algorithms include simulated annealing (Wu et al., 2011), tabu search (Bianchessi et al., 2007), genetic algorithm (Mansour et al., 2010), ant colony optimization (Wu et al., 2012, 2013), etc. In the observation scheduling of unmanned aerial vehicle (UAV), researchers often convert planning problems into classical models, such as traveling salesman problem model (Matthew, 2002), vehicle routing problem model (Brown, 2001) and multi-targets optimization model (Tian et al., 2006). At the same time, different intelligent optimization algorithms are used to solve the UAV scheduling problem. In recent years, with the serious situation of major unconventional emergencies at home and abroad, only a few papers have studied the problem of task planning under emergency conditions (Deng et al., 2014; Xue et al., 2015; Hu et al., 2016), but few published studies have comprehensively considered the problem of collaborative planning between different observation resources in disaster emergency.

Combining the disaster information acquired at the first time after the disaster and the dynamic simulation result of the disaster chain evolution process, this paper designed a collaborative monitoring resource planning system that considered high-medium-low orbit multi-satellites collaboration, aerospace and aviation collaboration, remote sensing and station collaboration, multi-temporal-spatial resolution combined and multi-sensor complementary advantages to ensures rapid acquisition of disaster site data.

\section{THE DESCRIPTION OF CO-PLANNING PROBLEMS}

\subsection{Observing Tasks and Observation Resources Analysis}

Task scheduling can be seen as the process of allocating limited resources to different tasks over time (Barbulescu et al., 2004). In collaborative earth observation systems, observation tasks and resources are important components. Before designing the collaborative planning of earth observation resources under the

\footnotetext{
* Corresponding author
} 
background of disaster emergency, observation tasks and resources are firstly analyzed.

The major disaster emergency observation task is different from conventional observation task. According to the types of disasters, observation tasks have different requirements for sensor types, target types, observation time, observation environment, etc. For example, for the observation task of flood, the flood inundation area can be regarded as regional targets for observation. Usually, the area target can't be completely observed by sensors at one time. Before taking the co-planning of multiple observation resources into account, the area target can be divided into strips or grids according to the characteristics of the observation resources. In addition, when the flood occurs, the observation area is mostly covered by cloud and rain. In this case, if the optical sensor cannot obtain effective images, the synthetic aperture radar (SAR) sensor observation is required, and if the continuous change of the flood is concerned, the periodic observation may be required in terms of observation time.

When the disaster occurs, one kind of observation resource is often difficult to meet observation needs, and multiple observation resources are required for effective planning and scheduling. In this paper, three different types of observation resources are considered: satellites, UAVs, and ground stations. The satellite observation has the advantages of not being restricted by regional and national boundaries and having wide observation range, but the acquisition and effectiveness of images are limited by observation environment conditions, time window constraints, and revisit cycles. The UAV observation has strong maneuverability and sensitivity, but the observation range is limited. The ground station observation is relatively stable, but the station distribution is discrete, so it is difficult to obtain the data of large area.

\subsection{The Description of Collaborative Planning Process}

The process of collaborative planning for major disasters is shown in Figure 1. After the disaster emergency response starts, the collaborative planning task will be started. According to the specific conditions of the disaster, firstly, the transit analysis of available satellite resources will be carried out to clarify the coverage of all satellite resources to the disaster area. For autonomous and controllable satellite resources of highmedium-low orbits, multi-temporal-spatial resolutions and multi-sensors, the maximum observation range of disaster areas is realized through observation task adjustment and observation instruction compilation. At the same time, the International Charter Space and Major Disasters (CHARTER, for short) is used to coordinate other available satellite resources for assisting observation. In the process of planning and scheduling, if there are no available satellite resources in the target area, collaborative observation planning of aerial remote sensing resources and station resources will be initiated immediately. Through fixed-point monitoring and spatial sampling monitoring, rapid acquisition of the disaster core site can be realized.

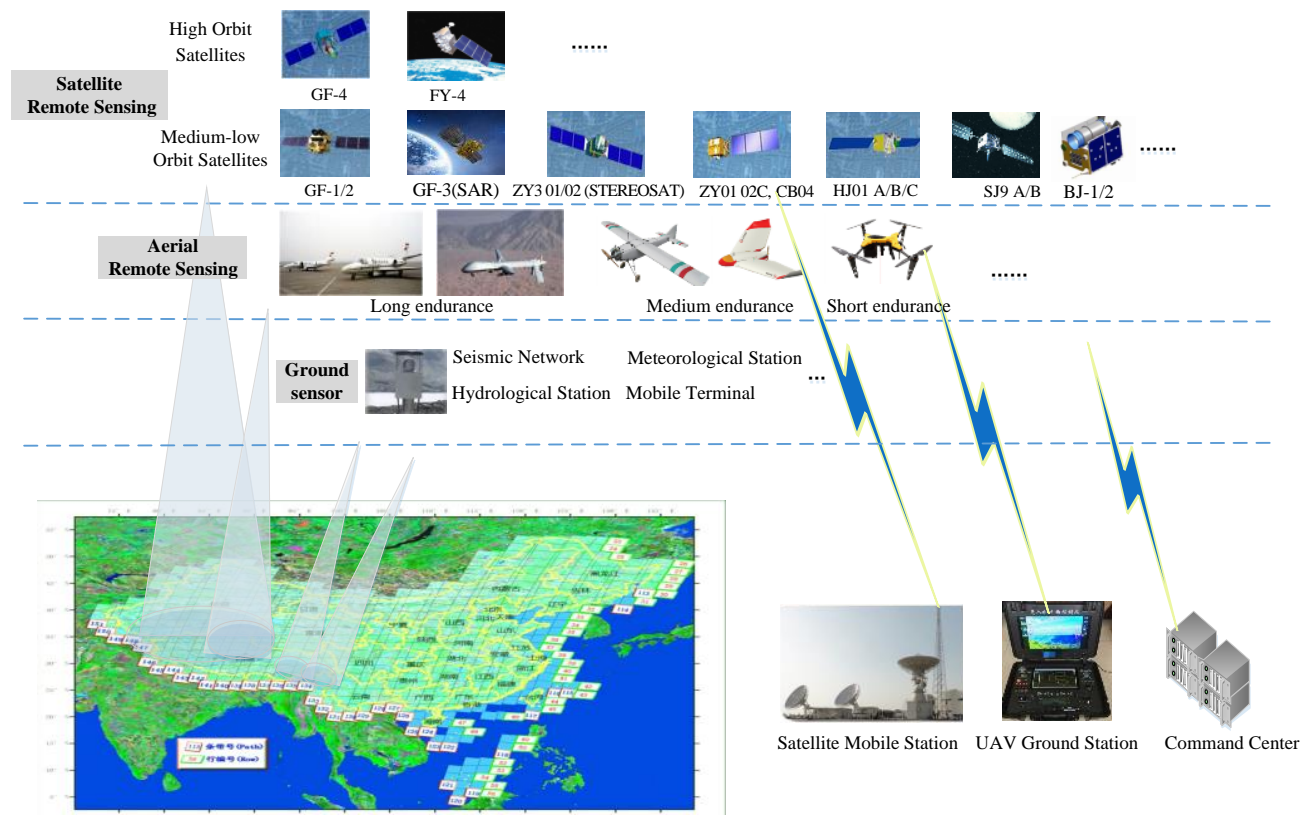

Figure 1. The process of co-planning

\section{OVERALL DESIGN OF COLLABORATIVE OBSERVATION PROJECT}

According to the characteristics and needs of major disaster observation tasks, the overall design of spaceborne, airborne and ground observation resources co-planning is carried out (Figure 2), mainly according to the disaster response level requirements after the disaster, to complete the observation resources task planning. Firstly, import the basic disaster information and disaster chain simulation results, and confirm the response level based on the disaster level. Secondly, when the task allocation project is formulated, the preliminary task allocation project is determined according to the analysis result of the observation resource catalogue system and the disaster rough estimation information. Thirdly, according to the disaster evolution information update and dynamic planning algorithm, local repair is completed, and the synthetic observation project is corrected. Finally, the optimal collaborative observation project is outputted. 


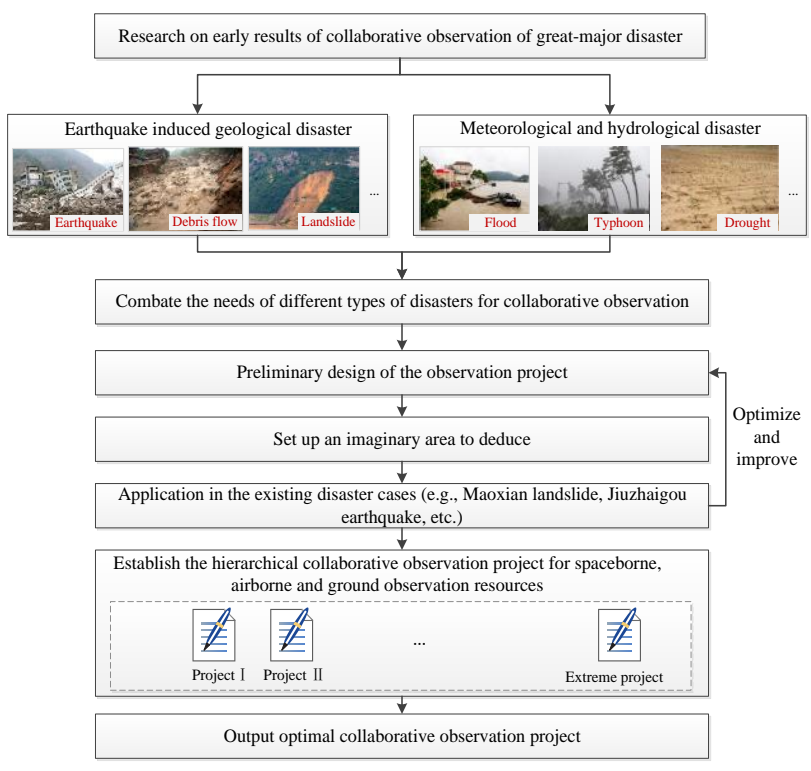

Figure 2. Overall design of collaborative observation project

\section{KEY TECHNOLOGY ANALYSIS AND DESIGN}

\subsection{The Design of Workflow Task Planning Corresponding to Disaster Respond Level}

According to the disaster response level requirements after the disaster, for different levels of disasters, the task response of the united scheduling of the corresponding level observation resources is performed, and a four-level workflow task planning system (Table 1) is designed, which are respectively the highest level, the second level, the priority level, and the ordinary level. Different levels of workflow task planning correspond to different observation resources scheduling. On the basis of fully analyzing the existing and future schedulable spaceborne, airborne and ground observation resources, high-medium-low orbit, multi-temporal-spatial resolution satellites, UAVs with different endurance capacities and different types of stations are effectively used, the coverage priority levels of disaster relief key monitoring regions and general regions are defined, and the observation data is timely provided to support for disaster site monitoring and disaster evolution monitoring and assists in decision-making.

\begin{tabular}{|c|c|c|c|}
\hline Response level & Task response & Observation mode response & Observation resource response \\
\hline Highest level & $\begin{array}{l}\text { Cancel all other observation } \\
\text { tasks and conduct emergency } \\
\text { observation of disaster }\end{array}$ & $\begin{array}{l}\text { Use all observable modes } \\
\text { (including extreme side-sway } \\
\text { angle, front and rear views, etc.) }\end{array}$ & $\begin{array}{l}\text { Schedule all available satellites } \\
\text { (various payloads), UAVs and } \\
\text { stations }\end{array}$ \\
\hline Second level & $\begin{array}{l}\text { Cancel all other observation } \\
\text { tasks and conduct emergency } \\
\text { observation of disaster }\end{array}$ & $\begin{array}{l}\text { Use risk-controllable observation } \\
\text { modes (including normal side- } \\
\text { sway angle, front and rear views, } \\
\text { etc.) }\end{array}$ & $\begin{array}{l}\text { Schedule all available satellites } \\
\text { (various payloads), UAVs and } \\
\text { stations }\end{array}$ \\
\hline Priority level & $\begin{array}{l}\text { Cancel other ordinary } \\
\text { observation tasks and carry out } \\
\text { disaster emergency observations }\end{array}$ & Use normal observation mode & $\begin{array}{l}\text { Schedule Satellites, UAVs, and } \\
\text { stations that have high } \\
\text { observation efficiency }\end{array}$ \\
\hline Ordinary level & Normal transit observation & Use normal observation mode & Normal scheduling \\
\hline
\end{tabular}

Table 1. The workflow tasks planning of different response levels

\subsection{Task Collaborative Planning Model and Algorithm Design}

Unlike conventional tasks, disaster emergency observation tasks reflect time urgency and therefore existing implementation programmes need to be inserted, which may need to be adjusted due to observation or resource constraints, often using a dynamic programming model (Jiang et al., 2013). Considering that different satellites have different maneuverability and orbits different UAVs have different endurance and operating efficiencies, different ground stations have different attributes and coverage, and different disaster response levels have different requirements for observation resources and task planning, a joint scheduling constraint planning model of spaceborne, airborne and ground remote sensing detecting resource driven by the emergency response task of major disasters was constructed.

The study of the planning and scheduling algorithms for earth observation tasks mainly focuses on deterministic algorithm and nondeterministic algorithm. The nondeterministic algorithm mainly includes heuristic algorithm and search algorithm. The deterministic algorithm is usually only suitable for solving small-scale problems. In the face of large-scale solutions, the nondeterministic algorithm is mainly used, and the search algorithm is more widely used. The search algorithm is divided into general search algorithm and intelligent optimization search algorithm. The key of general search algorithm is the design of search strategies. The design of intelligent search algorithm is more complex, and some problems need to be transformed to design such algorithm. The general search algorithm is simple in design, fast in solving speed, suitable for large-scale problems, and has the disadvantages of low search efficiency and easy to fall into local optimum. The intelligent search algorithm has strong universality, does not depend on specific problems, has high search efficiency and inherent parallel search ability, and is suitable for solving large-scale problems. If only one solution algorithm is used, precocity and local optimality are easy to occur, so the combined intelligent search algorithm is often used.

The problem of satellite scheduling has been proved to be an nondeterministic polynomial hard (NP-hard) problem. In order to meet the requirements of disaster emergency response, the satellite planning with high-medium-low orbits, multi-payloads, and multi-temporal-spatial resolutions needs to be considered, which will lead to the rapid increase of optimization solution space and face the problem of large-scale solution. The 
observation problem can be decomposed into task allocation and task collaboration, and the decomposition optimization strategy is used to solve the combined optimization problem of observation task allocation and collaboration. The algorithm structure is shown in Figure 3. The task allocation selects observation resources and time window for task based on disaster emergency level. According to the allocation plan, task collaboration divides satellite task assigned into groups based on orbit circles to optimally synthesis. At the same time, the results are repaired, and failed synthetic tasks are attempted to be added to the synthetic observation windows of other satellites. Finally, the synthetic observation project under this allocation scheme is obtained, and the results are fed back to guide the search process of the algorithm.

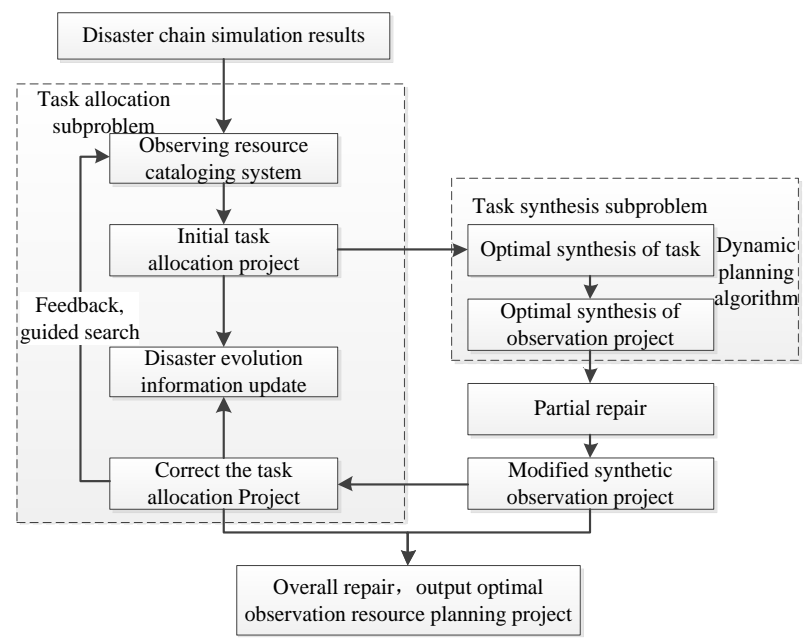

Figure 3. The algorithm structure based on decomposition optimization

\section{CONCLUSION}

In this paper, based on the co-planning requirements of spaceborne, airborne and ground observation resources under disaster emergency conditions, the characteristics of disaster emergency observation task and spaceborne, airborne and ground observation resources were analyzed, a joint scheduling and collaborative observation project for spaceborne, airborne and ground observation resources was designed, and the workflow task planning and collaborative observation algorithm optimization involved in observation project were analyzed and designed. The above research provided reference for disaster collaboration monitoring and emergency response service mechanisms. The research in this paper is preliminary. The disaster emergency observation is complicated and there are many uncertainties. The next step is to establish an extensible cataloguing system for observation resources, develop a visual simulation system suitable for the task planning of spaceborne, airborne and ground observation resources, apply the research results in the actual cases of major disasters to verify, and constantly optimize the collaborative observation project.

\section{ACKNOWLEDGEMENTS}

This research was supported by National Key R\&D Program of China (NO. 2017YFB0504101)

\section{REFERENCES}

Barbulescu L., Howe A. E., Whitley L. D., and Roberts M., 2004. Trading Places: How to schedule more in a Multiresource oversubscribed Scheduling problem. In: Fourteenth International Conference on Automated Planning and Scheduling, Whistler, British Columbia, C A, pp. 227-234.

Bianchessi N., Cordeau J. F., and Desrosiers J., 2007. A heuristic for the multi-satellite, multi-orbit and multi-user management of Earth observation satellites. European Journal of Operational Research, 177(2), pp. 750-762.

Brown D. T., 2001. Routing unmanned aerial vehicles while considering general restricted operating zones: AFIT/GOR/ENS/01M-04. In: Wright-Patterson: Air Force Institution of Technology.

Deng R., Tang H., Shan Y., Niu X. N., and Liu Y. H., 2014. Emergency task oriented satellite robust mission planning model and algorithm. Remote Sensing Information, 29(5), pp. 25-31.

Frank J., Jonsson A., Morris R., and Smith D., 2000. Planning and scheduling for fleets of earth observing satellites, In: Proceedings of the 6th International Symposium on Artificial Intelligence, Robotics, Automation and Space, pp. 1-8.

Gabrel V., 2006. Strengthened 0-1 linear formulation for the daily satellite mission planning. Journal of Combinato-rial Optimization, 11(4), pp. 341-346.

Gabrel V., and Vanderpooten D., 2002. Enumeration and interactive selection of efficient paths in a multiple criteria graph for scheduling an earth observing satellite. European Journal of Operational Researth, 139(3), pp. 533-542.

Hu C. L., Liu Y. D., and Zhang X., 2016. An emergency mission oriented evaluating method for space-based satellite sensors' observation combination. Geomatics and Information Science of Wuhan University, 41(10), pp. 1313-1318.

Jiang W., Hao H. C., and Li Y. J., 2013. Review of task scheduling research for the earth observing satellites. Systems Engineering and Electronics, 35(9), pp. 1878-1885.

Mansour M. A. A., and Dessouky M. M., 2010. A genetic algorithm approach for solving the daily photograph selection problem of the SPOT5 satellite. Computers \& Industrial Engineering, 58(3), pp. 509-520.

Matthew H., 2002. A method for estimating range requirements of tactical reconnaissance UAVs. In: AIAA's $1 s t$ Technical Conference and Workshop on Unmanned Aerospace Vehicles, Reston: AIAA, pp. 120-124.

Tian J., Shen L., and Zhang Y., 2006. Cooperative design, visualization, and engineering. Berlin: Springer Berlin Heidelberg, pp. 99-106.

Verfaillie G., Lemaitre M., and Schiex T., 1996. Russian doll search for solving constraint optimization problems, In: Proceedings of the National Conference on Artificial Intelligence, pp. 181-187. 
Wu G. H., Liu J., Ma M. H., and Qiu D S., 2013. A two-phase scheduling method with the consideration of task clustering for earth observing satellites. Computers \& Operations Research, 40(7), pp. 1884-1894.

Wu G. H., Ma M. H, Wang H. L., and Qiu D. S., 2011. Multisatellite observation scheduling based on task clustering. Acta Aeronautica ET Astronautica Sinica, 32(7), pp. 1275-1282.

Wu G. H., Ma M. H., Zhu J. H., and Qiu D. S., 2012. Multisatellite observation integrated scheduling method oriented to emergency tasks and common tasks. Journal of Systems Engineering and Electronics, 23(5), pp. 723-733.

Xue Z. J., Yang Z., Li J., and Zhao B., 2015. Autonomous mission planning of satellite for emergency. Command Control \& Simulation, 37(1), pp. 24-30. 